\title{
Archéopages
}

Archéopages

Archéologie et société

\section{Constructions en terre du Néolithique moyen près des rives de la Manche}

Catherine Bizien-Jaglin, Luc Laporte, Jean-Noël Guyodo et Julia Wattez

\section{(2) OpenEdition}

1 Journals

Édition électronique

URL : https://journals.openedition.org/archeopages/1133

DOI : 10.4000/archeopages. 1133

ISSN : 2269-9872

Éditeur

INRAP - Institut national de recherches archéologiques préventives

Édition imprimée

Date de publication : 1 mars 2016

Pagination : 22-23

ISSN : $1622-8545$

\section{Référence électronique}

Catherine Bizien-Jaglin, Luc Laporte, Jean-Noël Guyodo et Julia Wattez, « Constructions en terre du Néolithique moyen près des rives de la Manche », Archéopages [En ligne], 42 | 04-07/2015, mis en ligne le 01 juillet 2017, consulté le 05 juin 2021. URL : http://journals.openedition.org/archeopages/ 1133 ; DOI : https://doi.org/10.4000/archeopages.1133

Ce document a été généré automatiquement le 5 juin 2021.

(c) Inrap 


\title{
Constructions en terre du Néolithique moyen près des rives de la Manche
}

\author{
Catherine Bizien-Jaglin, Luc Laporte, Jean-Noël Guyodo et Julia Wattez
}

1 À Lillemer (Ille-et-Vilaine), au fond de la baie du Mont-Saint-Michel, les vestiges d'une architecture en terre néolithique ont dernièrement été mis en évidence (Laporte et al., 2015) [ill. 1]. Il s'agit de bâtiments aux murs construits à base de terre crue, sans armature de bois, préludant aux aménagements d'une vaste enceinte du Néolithique moyen, au cours de la seconde moitié du v $v^{e}$ millénaire avant notre ère. Celle-ci cerne l'ensemble d'une butte rocheuse qui émerge des marais environnants, riches également de vestiges appartenant à cette période. La mise en évidence de constructions en terre est d'abord passée par l'identification de fragments isolés de pains de terre cuite par un incendie, puis d'autres en terre crue. Un parement de mur a été rendu identifiable par son contact vertical avec des niveaux incendiés et effondrés. Un autre niveau de lecture correspond à la prise en compte d'épais bourrelets d'argile, lors de la recherche des murs. Une étude micromorphologique a permis d'identifier la terre massive comme technique constructive, notamment sous la forme d'éléments modulaires mis en place par façonnage direct et préparés à partir de limons et de graviers issus des sols environnants ou des formations palustres [ill. 2]. La fouille des vestiges de murs conservés en élévation et le dégagement des sols se sont effectués au détriment des masses argileuses intercalées (murs effondrés, recharges successives de sol et/ou possibles éléments construits), tant la lecture de ce sédiment à première vue très homogène s'est avérée complexe. 
1. Des niveaux de sols étagés et des vestiges de constructions en terre, conservés en élévation, ont été mis au jour à Lillemer sous la masse du talus du Néolithique moyen.

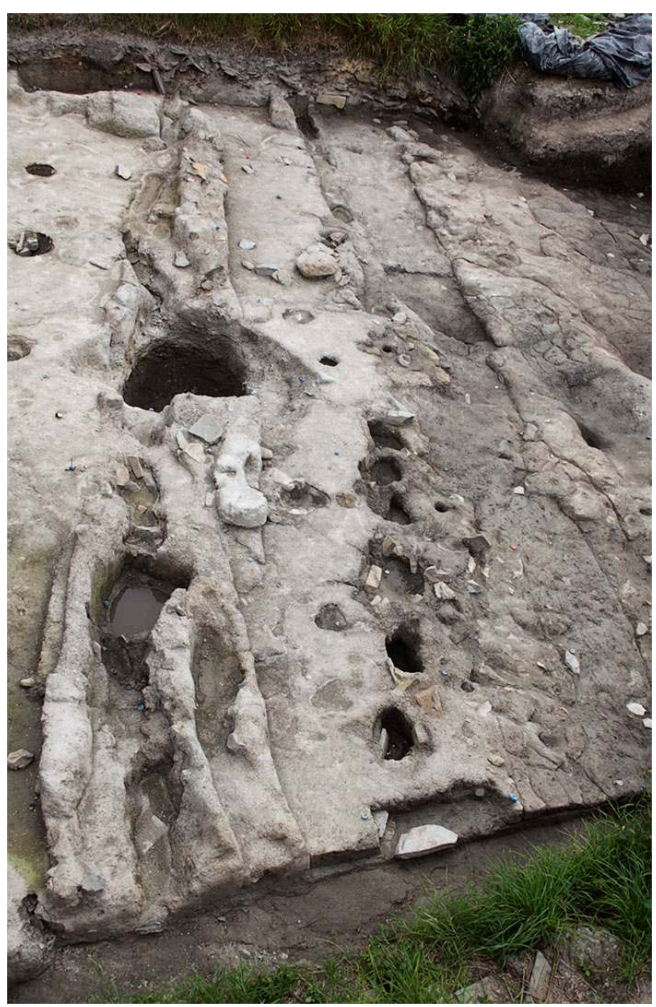

(c) L. Laporte

2. Micromorphologie mur ST 78, emboîtement de mottes façonnées, scan de lame mince.

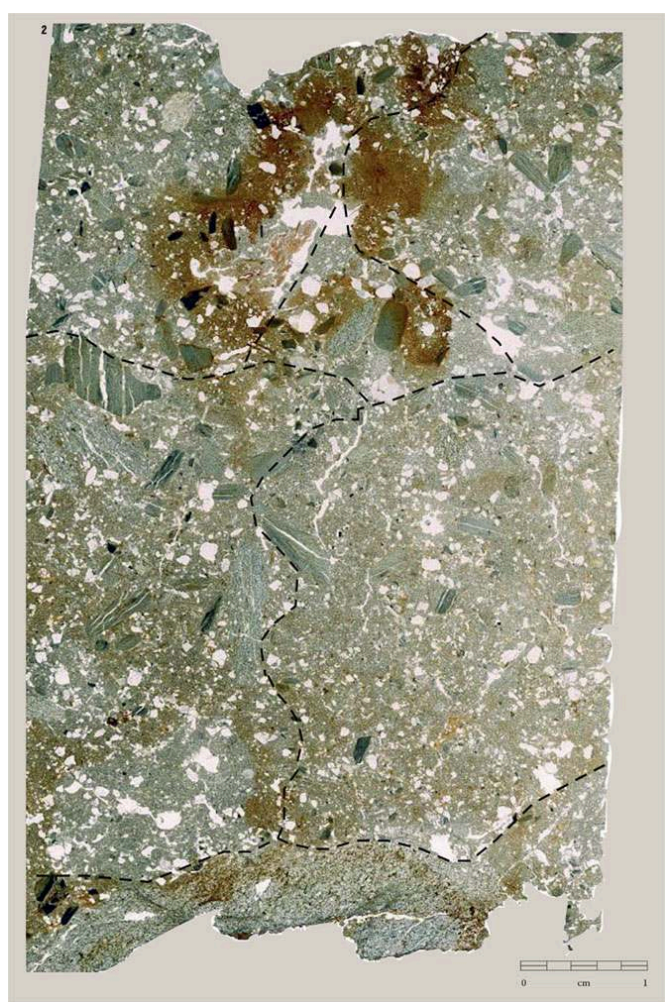

(C) J. Wattez, Inrap 
2 L'une des constructions mises au jour, large de 2,50 m à l'intérieur et longue d'au moins $9 \mathrm{~m}$, est délimitée par deux murs latéraux - nord et sud - larges de 1,20 m, un mur de refend à l'est apparemment de moindre puissance, un sol et quatre fosses d'implantation de poteaux suivant un axe décentré. Le mobilier y est particulièrement rare. On note l'absence de foyer. Des poutres de bois rubéfiées et des dalles de schiste brûlées témoignent, en lien avec les trous de poteau, d'une couverture - sans doute végétale - maintenue avec des dalles et soutenue par une charpente en bois. Au sud et légèrement en contrebas, un deuxième niveau de sol conservé sur une plus faible superficie a été percé par les aménagements nécessaires à la mise en place, postérieure, d'une forte palissade. Ce sol ne porte pas les altérations propres à un espace ouvert. également constitué de pains de terre, c'est-à-dire bâti, il présente les mêmes qualités que celui, interne, du bâtiment précédemment décrit. Plus au sud encore, un massif de 2,5 m de largeur est parallèle aux deux murs déjà mentionnés. Différentes natures de sédiments mis en œuvre, de même que les témoins de murs antérieurs repris, ou arasés, montrent l'existence de plusieurs états successifs. Le plan de ces constructions se rapproche de celui, aggloméré, d'un village plutôt que de celui des grands bâtiments sur poteaux porteurs habituellement connus pour cette période dans cette région.

Rétrospectivement, les témoins d'aménagements similaires utilisant la terre crue avaient déjà été observés en différents points de la butte de Lillemer lors de sondages et fouilles, tant programmées que préventives (Bizien-Jaglin et al., 2010 ; Laporte et al., 2014). Sur le flanc sud de la butte, une épaisse couche argileuse contenant des artefacts néolithiques a été dégagée, avant la mise en évidence de fronts de taille résultant de la construction de terrasses étagées. L'épaisseur de cet horizon et la restitution des reliefs rocheux originaux orientent vers des apports anthropiques importants. Cette hypothèse a également été confirmée par les analyses micromorphologiques. Un peu plus bas sur cette pente, une coupe montrait une forme rectangulaire évoquant une possible élévation de terre construite. Au nord de la butte, le rempart observé en coupe est entièrement constitué de ces mêmes matériaux, recouvrant là aussi des reliefs antérieurs en argile. L'ensemble de ces observations montre que les constructions étudiées dans la parcelle 733 (qui recoupe le tracé du talus), sur une superficie de seulement $150 \mathrm{~m}^{2}$, ne constituent pas des éléments isolés. Elles résultent d'énormes investissements humains, à l'image de ce que suggérait déjà l'aménagement de terrasses sur les flancs de la butte ou, plus tard encore, l'édification d'un talus périphérique ceinturant l'ensemble de la butte rocheuse sur plus d'un kilomètre linéaire. Ces fouilles ont aussi contribué à attirer l'attention - jusque sur les rives de la Manche et pour une période aussi ancienne que le Néolithique moyen - sur une matière omniprésente sur les chantiers de fouilles, l'argile, que tout le monde sait implicitement avoir été largement utilisée pendant les périodes anciennes, sans toujours penser à mettre en évidence son usage comme matériau de construction. 


\section{BIBLIOGRAPHIE}

BIZIEN-JAGLIN C., GUYODO J.-N., LAPORTE L., 2010, « Occupation du Néolithique moyen autour de la butte de Lillemer (Ille-et-Vilaine) », Archéo-Théma, 101, septembre 2010, p. 26-34.

LAPORTE L., BIZIEN-JAGLIN C., GUYODO J.-N., 2014, « Enceintes néolithiques de l'ouest de la France : une archéologie des fossés ? », in JOUSSAUME R., LARGE J.M. (ÉD.), Enceintes néolithiques de l'ouest de la France, de la Seine à la Gironde, Actes du Colloque CrabeNéo, sept. 2012, Quimper, Association des Publications Chauvinoises, p. 455-488.

LAPORTE L., BIZIEN-JAGLIN C., WATTEZ J. et al., 2015, « Another brick in the wall: fifth millennium BC earthen-walled architecture on the Channel shores ", Antiquity, 89, 346, p. 800-817.

\section{AUTEURS}

\section{CATHERINE BIZIEN-JAGLIN}

Centre régional d'archéologie d'Alet

\section{LUC LAPORTE}

CNRS, UMR 6566 « CReAAH »

JEAN-NOËL GUYODO

Université de Nantes, UMR 6566 « CReAAH »

\section{JULIA WATTEZ}

Inrap, UMR 5140, « Archéologie des Sociétés Méditerranéennes » 